\title{
Association of systemic immune-inflammation index (SII) with presence of isolated coronary artery ectasia
}

Aydın Rodi Tosu, Halil Ibrahim Biter

$1^{\text {st }}$ Cardiology Department, Haseki Training and Research Hospital, University of Health Sciences Turkey, Istanbul, Turkey

Submitted: 31 March 2021

Accepted: 21 July 2021

Arch Med Sci Atheroscler Dis 2021; 6: e152-e157

DOI: https://doi.org/10.5114/amsad.2021.109253

Copyright $\odot 2021$ Termedia \& Banach

\section{Abstract}

Introduction: The systemic immune-inflammation index (SII) has been developed based on the calculation of $\mathrm{N} \times \mathrm{P} / \mathrm{L}$ (N, P and $L$ represent neutrophil count, platelet count and lymphocyte count, respectively), and it is widely used as a marker of inflammation and an indicator of cardiovascular outcomes in patients with coronary artery disease. We examined a possible association between SII and the presence of isolated coronary artery ectasia (CAE).

Material and methods: In this retrospective case-control study, a total of 4400 patients who underwent elective coronary angiography between June 2015 and July 2020 were retrospectively screened. Following the application of exclusion criteria, our study population consisted of 139 CAE patients and 141 age- and gender-matched subjects who proved to have normal coronary angiograms.

Results: The median value of SII was found to be statistically significantly higher in patients with CAE $(p<0.01)$. SII level $\geq 809$ measured on admission had $48 \%$ sensitivity and $82 \%$ specificity in predicting isolated CAE in ROC curve analysis. In this ROC analysis, the predictive powers of neutrophil-to-lymphocyte ratio (NLR) and SII in determining the presence of ectasia were compared, and the predictive power of SII was significantly stronger than $\mathrm{N} / \mathrm{L}$ ratio $(p=0.003)$. In the multivariate analysis, hyperlipidaemia $(O R=1.978,95 \% \mathrm{Cl}: 1.168-3.349, p=0.01)$, smoking $(O R=1.86$, $95 \% \mathrm{Cl}: 1.090-3.127, p=0.023) \mathrm{N} / \mathrm{L}$ ratio $(\mathrm{OR}=1.192,95 \% \mathrm{Cl}:(1.114-$ 1.997, $p=0.07)$ and SII (OR $=1.002,95 \% \mathrm{Cl}: 1.001-1.003, p<0.01)$ were independent predictors of the presence of isolated CAE.

Conclusions: SII is a readily available clinical laboratory value that is associated with the presence of isolated CAE. Our findings may indicate a common pathophysiological mechanism between CAE and coronary artery disease.

Key words: systemic immune inflammation index (SII), inflammation, coronary artery ectasia.

\section{Introduction}

The term ectasia was first used by Bjork [1]. Markis et al. [2] performed the first prospective evaluation of incidence of coronary artery aneurysm. Coronary ectasia is defined as localised or diffuse dilatation of the coronary artery $\geq 1.5$ times the adjacent normal vessel segment [3].

Although the underlying mechanisms are not fully understood, coronary artery ectasia (CAE) is considered to be an original form of vascular remodelling in response to atherosclerosis. However, it is not clear why some patients develop CAE while most do not. In previous histopatho-

\author{
Corresponding author: \\ Aydın Rodi Tosu \\ $1^{\text {st }}$ Cardiology \\ Department \\ Haseki Training and \\ Research Hospital \\ University of \\ Health Sciences \\ Istanbul, Turkey \\ E-mail: aydinroditosu@gmail. \\ com
}


logical studies, elastin, collagen, and extracellular matrix damage and inflammatory cell accumulation have been reported in ectatic arteries [4-6].

In recent years, there has been increasing interest in describing a simple inflammatory marker in order to facilitate early recognition of patients who may have an increasing risk for future cardiovascular disease. Recently, the systemic immune inflammation index (SII) has been developed based on the calculation of $N \times P / L$ (N, P and $L$ represent neutrophil count, platelet count and lymphocyte count, respectively) to consider patients' inflammatory and immune status simultaneously. High SII has been reported to be associated with poor outcomes in cancer patients [7].

As inflammation plays a significant role in the development of CAE, we hypothesized that there may be a relation between SII and CAE. Hence, in the present study, we aimed to evaluate the potential utility of SII in predicting CAE.

\section{Material and methods}

\section{Study population}

In this retrospective case-control study, a total of 4400 patients who underwent elective coronary angiography between June 2015 and July 2020 were retrospectively screened. After evaluation regarding exclusion criteria, 139 (3.1\%) patients were found to have an isolated CAE. Exclusion criteria were as follows: I) evidence of acute or chronic infection, II) systemic inflammatory or autoimmune disease, III) a history of any liver disease (with liver function parameters more than three times the upper normal limit), IV) any clinically significant endocrine, hematologic, respiratory, or metabolic diseases, V) malignancy. VI) acute coronary syndrome.

The control group consisted of 141 angiographically normal coronary artery patients who matched this group in terms of age, gender, and body mass index. Baseline demographic characteristics and related clinical information were retrieved from the hospital's electronic database. Our local ethics committee approved the study protocol in accordance with the principle of the Declaration of Helsinki. Informed consent was waived because this study had a retrospective design.

\section{Laboratory analysis}

In our hospital, blood samples are collected from the antecubital vein by an atraumatic puncture prior to the coronary angiography and sent to the laboratory for analysis within 1 hour after collection. Venous blood was collected in a tube containing K3 EDTA for measurement of haematological indices in all patients undergoing coro- nary angiography. Haematological indices were evaluated from CBC (complete blood count) analysis performed by a Coulter LH 780 Hematology Analyzer (Beckman Coulter Ireland Inc. Mervue, Galway, Ireland).

\section{SII}

In all patients, blood samples were collected from the antecubital vein before coronary angiography. Complete blood cell counts, including platelet, neutrophil, and lymphocyte, were determined with an auto-analyser. The SII was calculated as total peripheral platelet count $(\mathrm{P}) \times$ neutrophil-to-lymphocyte ratio $(\mathrm{N} / \mathrm{L})(\mathrm{SII}=\mathrm{P} \times \mathrm{N} / \mathrm{L}$ ratio $)[7]$.

\section{Coronary angiography}

In the current study, the patients who were diagnosed with typical angina or those with a suspected or positive finding in one of the non-invasive methods that is performed for detection of coronary ischemia underwent elective coronary angiography. Coronary angiography was performed via the femoral or radial artery according to Judkins's technique. All coronary angiograms were recorded into DICOM digital media with a rate of 25 frames/ms. All coronary angiograms were evaluated by two experienced interventional cardiologists who were blinded to the patient's clinical data.

\section{Definitions}

The CAE was defined as proposed in a previous study [8]. Hypertension was defined as a systolic blood pressure of $140 \mathrm{mmHg}$ or higher, diastolic blood pressure of $90 \mathrm{~mm}$ or higher or use of an antihypertensive medicine [9]. The presence of diabetes mellitus was defined as fasting blood glucose of $\geq 126 \mathrm{mg} / \mathrm{dl}$ or higher or current use of antidiabetic treatment or being on a diet [10].

\section{Statistical analysis}

Continuous variables were presented as means \pm standard deviations if normally distributed and medians (interquartile ranges (IQRs)) if not normally distributed, while categorical variables were given as percentages. The chi-squared $\left(\chi^{2}\right)$ test was used to compare categorical variables between the groups, while the Kolmogorov-Smirnov test was employed to assess whether the variables were normally distributed. Student's $t$-test or the Mann-Whitney $U$ test was used to compare the continuous variables between the groups according to whether they were normally distributed or not. Variables found to be associated at a level of $p<0.05$ according to the univariate analysis were included in the multivariate logistic regression 
Table I. Demographic distribution and laboratory findings of all patients

\begin{tabular}{|lcccc|}
\hline Variables & $\begin{array}{c}\text { All population } \\
(n=280)\end{array}$ & $\begin{array}{c}\text { Ectasia(-) } \\
(n=141)\end{array}$ & $\begin{array}{c}\text { Ectasia(+) } \\
(n=139)\end{array}$ & $P$-value \\
\hline Male gender, $n(\%)$ & $119(42.5)$ & $60(42.6)$ & $59(42.4)$ & 0.986 \\
\hline Age & $56.9 \pm 8.0$ & $57.9 \pm 8.8$ & $55.9 \pm 7.0$ & 0.035 \\
\hline BMI & $25.3 \pm 1.3$ & $25.2 \pm 1.3$ & $25.4 \pm 1.3$ & 0.235 \\
\hline Hypertension, $n(\%)$ & $158(56.6)$ & $78(55.3)$ & $80(57.6)$ & 0.706 \\
\hline Diabetes, $n(\%)$ & $168(60)$ & $86(61)$ & $82(59)$ & 0.733 \\
\hline Smoking, $n(\%)$ & $106(37.9)$ & $44(31.2)$ & $62(44.6)$ & 0.021 \\
\hline Hyperlipidaemia, $n(\%)$ & $114(40.7)$ & $43(30.5)$ & $71(51.1)$ & $<0.001$ \\
\hline Creatinine [mg/dl] median [IQR] & $0.8[0.8-1.0]$ & $0.8[0.9-1.1]$ & $0.8[0.8-0.9]$ & 0.945 \\
\hline CRP [mg/dl] median [IQR] & $0.9[0.6-1.5]$ & $0.9[0.6-1.5]$ & $1.1[0.6-1.6]$ & 0.507 \\
\hline Haemoglobin [g/dl] & $13.8 \pm 2.4$ & $13.9 \pm 2.1$ & $13.6 \pm 2.7$ & 0.174 \\
\hline Neutrophil, median [IQR] & $2.47[1.25-3.92]$ & $1.27[0.7-2.32]$ & $3.76[2.73-5.59]$ & $<0.001$ \\
\hline Lymphocyte, median [IQR] & $1.14[0.55-1.49]$ & $0.57[0.31-1.0]$ & $1.45[1.2-1.88]$ & $<0.001$ \\
\hline Platelet & $300.3 \pm 55.2$ & $282.5 \pm 39.3$ & $318.9 \pm 63.5$ & $<0.001$ \\
\hline NLR, median [IQR] & $2.3[1.9-3.1]$ & $2.2[1.9-2.5]$ & $2.6[2.1-3.3]$ & 0.001 \\
\hline SII, median [IQR] & $667[553-893]$ & $617[513-772]$ & $768[600-1132]$ & $<0.001$ \\
\hline
\end{tabular}

CRP - C-reactive protein, NLR - neutrophil/lymphocyte ratio, SII - systemic immune-inflammation index, BMI - body mass index.

analysis with the results reported as the odds ratios (OR) and 95\% confidence intervals ( $\mathrm{Cl})$. The ability of NLR and SII to determine the presence of coronary ectasia was analysed using the receiving operating characteristic (ROC) curve and area under the curve (AUC), accompanied by the 95\% confidence interval. The optimal cut-off value was also calculated from the point of maximal sensitivity and specificity by using Youden's index. To compare the predictive performance of the CAR, CRP and albumin the test of DeLong et al. was also used. The threshold of statistical significance was established at $p<0.05$. All statistical analyses were performed using the software SPSS Statistics version 24.0 (IBM Corp., Armonk, NY, USA). ROC curves of the models were compared using the MEDCALC software (Software bvba 13, Ostend, Belgium).

\section{Results}

The mean age of study population was 55.9 \pm 7.0 years and 59 (42.4\%) patients were male.

Table II. Vascular involvement and extent of ectatic vessels

\begin{tabular}{|c|c|}
\hline \multicolumn{2}{|c|}{ Distribution of ectasia: } \\
\hline LAD & $28(19.8 \%)$ \\
\hline$C X$ & $36(25.5 \%)$ \\
\hline RCA & $83(58.8 \%)$ \\
\hline \multicolumn{2}{|c|}{ Number of ectatic vessels: } \\
\hline One vessel & $65(46.1 \%)$ \\
\hline Two vessels & $45(31.9 \%)$ \\
\hline Three vessels & $31(21.9 \%)$ \\
\hline
\end{tabular}

The demographic and laboratory data of all patients are summarized in Table I. The prevalence of comorbidities, including hypertension, diabetes, and body-mass index (BMI) were not different between the groups. There were similarities in both groups between CRP and haemoglobin levels. Neutrophil, lymphocyte and platelet levels were significantly higher in the group with ectasia $(p<0.001)$.

Moreover, we found that isolated CAE most commonly affects the right coronary artery (58.8\%), left anterior descending artery (19.8\%), and circumflex artery (25.5\%) (Table II). When we divided the study population into two groups according to the SII value, diabetes, hyperlipidaemia and the presence of isolated CAE were significantly more frequent in the high SII group ( $p<0.001, p=0.04, p<0.001$, respectively, Table III). Also we found that smoking was more common in the ectasia(+) group $(31.2 \%$ vs. $44.6 \%$, $p=0.02$, Table I).

In the ROC curve analysis, SII value $>809$ was identified as an effective cut-off point in the segregation of the presence or absence of isolated CAE (AUC $=0.674,95 \% \mathrm{Cl}: 0.616-0.729, p<$ 0.001 ). SII value $>809$ yielded sensitivity of $42 \%$, specificity of $82 \%, \mathrm{~N} / \mathrm{L}$ ratio for detecting the presence of coronary ectasia (AUC $=0.628,95 \% \mathrm{Cl}$ : $0.562-0.684 ; p<0.001)$. In this ROC analysis, the predictive powers of NLR and SII in determining the presence of ectasia were compared, and the predictive power of SII was statistically significantly stronger than NLR ( $p=0.003)$ (Figure 1).

Variables found to be statistically significant in univariate analyses were included in the mul- 
Table III. Baseline characteristics of patients with low and high SII

\begin{tabular}{|lccc|}
\hline Variables & Low SII $(n=187)$ & High SII $(n=93)$ & $P$-value \\
\hline Male gender, $n(\%)$ & $88(47.1)$ & $31(33.3)$ & 0.029 \\
\hline Age & $57.2 \pm 7.9$ & $56.3 \pm 8.3$ & 0.357 \\
\hline BMI & $25.4 \pm 1.3$ & $25.2 \pm 1.4$ & 0.160 \\
\hline Hypertension, $n(\%)$ & $111(59.4)$ & $47(50.5)$ & 0.161 \\
\hline Diabetes, $n(\%)$ & $125(66.8)$ & $43(46.2)$ & 0.001 \\
\hline Smoking, $n(\%)$ & $69(36.9)$ & $37(39.8)$ & 0.639 \\
\hline Hyperlipidaemia, $n$ (\%) & $65(34.8)$ & $49(52.7)$ & 0.004 \\
\hline Creatinine [mg/dl] median [IQR] & $0.8[0.8-1.0]$ & $0.8[0.8-1.0]$ & 0.434 \\
\hline CRP [mg/dl] median [IQR] & $0.9[0.6-1.5]$ & $1.0[0.8-1.6]$ & 0.989 \\
\hline Haemoglobin [g/dl] & $13.8 \pm 2.6$ & $13.8 \pm 2.2$ & 0.776 \\
\hline Neutrophil, median [IQR] & $2.1[0.8-2.72]$ & $4.48[3.36-6.75]$ & $<0.001$ \\
\hline Lymphocyte, median [IQR] & $1.06[0.39-1.3]$ & $1.4[1.1-1.9]$ & $<0.001$ \\
\hline Platelet & $280.3 \pm 36.6$ & $341.2 \pm 64.8$ & $<0.001$ \\
\hline NLR, median [IQR] & $2.1[1.9-2.4]$ & $3.4[2.84-4.1]$ & $<0.001$ \\
\hline SII, median [IQR] & $599[511-672]$ & $1139[896-1408]$ & $<0.001$ \\
\hline CAE, $n$ (\%) & $40(28.3)$ & $141(71.6)$ & $<0.001$ \\
\hline
\end{tabular}

$C R P$ - C-reactive protein, $R C A$ - right coronary artery, $C X$ - circumflex artery, $L A D$ - left anterior descending artery, NLR - neutrophil/ lymphocyte ratio, SII - systemic immune-inflammation index.

tivariate logistic regression analysis (Table IV). In the multivariate analysis, hyperlipidaemia (OR $=1.978,95 \% \mathrm{Cl}: 1.168-3.349, p=0.01)$, smoking $(\mathrm{OR}=1.86,95 \% \mathrm{Cl}: 1.090-3.127, p=0.023)$ $\mathrm{N} / \mathrm{L}$ ratio $(\mathrm{OR}=1.192,95 \% \mathrm{Cl}: 1.114-1.997$, $p=0.07)$ and $\mathrm{SII}(\mathrm{OR}=1.002,95 \% \mathrm{Cl}: 1.002-$ $1.003, p<0.01)$ were independent predictors of the presence of isolated CAE.

\section{Discussion}

This is the first study evaluating the relationship between SII and the presence of isolated CAE. As a simple and easily obtained haematological parameter from complete blood count, SII may be used in predicting risk of CAE.

In previous publications, CAE was found in 1.5$5 \%$ of patients included in coronary angiographic studies [11]. In this study, we reported an incidence of isolated CAE of $3.1 \%$ among patients who underwent coronary angiography for the presence of chest pain or had objective signs of ischaemia.

Coronary atherosclerosis is present in almost $50 \%$ of patients with CAE, suggesting a common causative mechanism in the formation of both coronary artery disease (CAD) and CAE. Previously, inflammatory markers such as CRP, interleukin-6, and vascular adhesion molecules were investigated in patients with CAE [12]. A recent study by Li et al. showed that interleukin- 6 and CRP were significantly higher in patients with CAE than in patients with normal coronary arteries [13]. In our study, there was no significant difference in CRP levels in low and high SII groups. The importance of NLR in cardiovascular disease has been

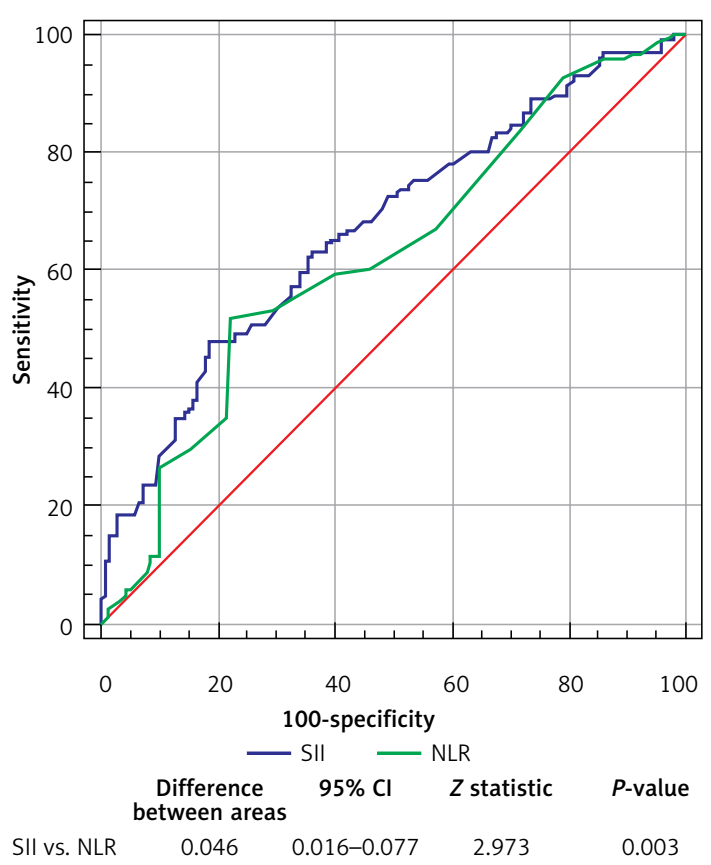

Figure 1. ROC curves of the SII (blue) and NLR (green) scores for detecting the presence of coronary ectasia

investigated in a few studies. Işık et al. found that a greater baseline NLR value was independently associated with the presence of isolated CAE [14]. Kundi et al. reported that high monocyte/HDL ratio (MHR) is an independent predictor of CAE and obstructive coronary artery disease [15].

The associations between CAE and inflammatory vasculitides such as Kawasaki disease, Takayasu arteritis, Behçet's disease, and connec- 
Table IV. Two different adjusted multivariate Cox proportional risk regression analysis models to determine the predictors of coronary ectasia

\begin{tabular}{|lcccc|}
\hline Variables & \multicolumn{2}{c}{$\begin{array}{c}\text { Model 1 } \\
\text { Multivariate }\end{array}$} & \multicolumn{2}{c|}{$\begin{array}{c}\text { Model 2 } \\
\text { Multivariate }\end{array}$} \\
\cline { 2 - 5 } & OR $(95 \% \mathrm{Cl})$ & P-value & OR $(95 \% \mathrm{Cl})$ & $P$-value \\
\hline Age & $0.971(0.941-1.002)$ & 0.067 & $0.972(0.941-1.004)$ & 0.067 \\
\hline Smoking & $1.722(1.030-2.880)$ & 0.038 & $1.846(1.090-3.127)$ & 0.023 \\
\hline Hyperlipidaemia & $2.348(1.413-3.903)$ & 0.001 & $1.978(1.168-3.349)$ & 0.011 \\
\hline NLR & $1.492(1.114-1.997)$ & 0.007 & - & - \\
\hline SII & - & - & $1.002(1.001-1.003)$ & $<0.001$ \\
\hline
\end{tabular}

tive tissue diseases support the inflammatory origin of CAE [16]. Plasma E-selectin, intercellular adhesion molecule-1, and vascular cell adhesion molecule-1 were significantly higher in patients with isolated CAE [17]. In a study, higher levels of inflammatory markers including tumour necrosis factor $\alpha$ and interleukin 6 (IL-6) were increased in patients with CAE [18]. Furthermore, Turhan et al. [19] found that CRP, an indicator of the systemic inflammatory response, was also significantly higher in patients with isolated CAE than in those with a normal coronary artery. Accumulation of inflammatory cells in the medial layer of coronary arteries was also reported in histopathological examinations of patients with CAE [20, 21]. In addition, the levels of cellular adhesion molecules $C D 11 \mathrm{~b}$ and CD 45 were higher in patients with CAE [22].

Chronic inflammation has been considered to have major roles in several important diseases. Zhong et al. [7] first reported on the use of the SII in hepatocellular carcinoma, and the index had significant associations with prognostic clinical outcomes, including vascular invasion, tumour size and early recurrence. More and more clinical observation studies indicate that $\mathrm{SII}$ is a powerful tool for predicting survival outcomes in multiple solid cancers [23, 24]. Recently, Seo et al. [25] reported for the first time the predictive value of SII in patients with chronic heart failure. The current study further extended the importance of SII to cardiovascular disease. We found that the rate of development of ectasia is higher in smokers compared to non-smokers. Cigarettes accelerate atherosclerosis together with insecticides, toxic substances, the direct effect of some trace elements and the metabolic and biochemical disorders it causes. Oxidative stress caused by smoking plays an important role in the development of atherosclerosis. Radical oxidative products due to oxidative stress increase the use of antioxidant enzymes and lead to their reduction. The relationship between the reduction of these enzymes and mitochondrial DNA damage in the vessel wall is thought to play an important role in atherosclerosis and organ damage [26]. This physiopathological mechanism may explain the higher incidence of coronary ectasia in smokers.

Our results provided evidence that the inflammatory process caused by high SII levels may cause microvascular dysfunction in CAE patients. The SII is a simple and inexpensive marker of inflammation, and it can be calculated easily from complete blood count parameters. Elevated SII, which is a newly introduced inflammatory marker, may have predictive value for the prediction of CAE in daily clinical practice.

In conclusion, we observed that SII levels were higher in CAE patients than in healthy subjects. Our findings may indicate a common pathophysiological mechanism between CAE and coronary artery disease. Since there are common pathophysiological mechanisms between coronary ectasia and coronary artery disease, there is no specific treatment for coronary ectasia. There is no evidence that the treatment of coronary ectasia can reduce SII. Long-term prospective studies are needed. Finally, our findings warrant further studies to describe a clear role of this marker.

This study had some limitations. First, our study had a retrospective design. Second, we were not able to evaluate the plaque burden because patients without evidence of luminal narrowing shown by angiography may also have a plaque burden in the wall of the coronary vessels. Third, use of a spot laboratory value rather than values at a time interval may be another limitation because the development of CAE is a chronic condition. Finally, we did not evaluate other well-known inflammatory markers such as fibrinogen in the study.

\section{Conflict of interest}

The authors declare no conflict interest.

\section{References}

1. Bjork L. Ectasia of coronary arteries. Radiology 1966; 87: 33-4.

2. Markis JE, Joffe CD, Cohn PF, et al. Clinical significance of coronary arterial ectasia. Am J Cardiol 1976; 37: 217-22. 
3. Yilmaz H, Sayar N, Yilmaz M, et al. Coronary artery ectasia: clinical and angiographical evaluation. Turk Kardiyo Dern Ars 2008; 36: 530-5.

4. Diet F, Pratt RE, Berry GJ, Momose N, Gibbons GH, Dzau VJ. Increased accumulation of tissue ACE in human ath erosclerotic coronary artery disease. Circulation 1996; 94: 2756-67.

5. Tamarina NA, McMillan WD, Shively VP, Pearce WH. Expression of matrix metalloproteinases and their inhibitors in aneurysms and normal aorta. Surgery 1997; 122 264-71.

6. Knox JB, Sukhova GK, Whittemore AD, Libby P. Evidence for altered balance between matrix metalloproteinases and their inhibitors in human aortic diseases. Circulation 1997; 95: 205-12.

7. Zhong JH, Huang DH, Chen ZY. Prognostic role of systemic immune-inflammation index in solid tumors: a systematic review and meta-analysis. Oncotarget 2017; 8: 75381-8.

8. Markis JE, Joffe CD, Cohn PF, Feen DJ, Herman MV, Gorlin R. Clinical significance of coronary arterial ectasia. Am J Cardiol 1976; 37: 217-22.

9. Mancia G, Fagard R, Narkiewicz K, et al. 2013 ESH/ESC guidelines for the management of arterial hypertension: The Task Force for the Management of Arterial Hypertension of the European Society of Hypertension (ESH) and of the European Society of Cardiology (ESC). Eur Heart J 2013; 34: 2159-219.

10. Mancia G, De Backer G, Dominiczak A, et al. 2007 guidelines for the management of arterial hypertension: the task force for the management of arterial hypertension of the European Society of Hypertension (ESH) and of the European Society of Cardiology (ESC). J Hypertens 2007; 25: 1105-87.

11. Boles U, Eriksson P, Zhao Y, Henein MY. Coronary artery ectasia: remains a clinical dilemma. Coron Artery Dis 2010; 21: 318-20.

12. Sadr-Ameli $M$, Sharifi $M$. The natural history of ectatic coronary artery disease. Iranian Heart J 2001; 2: 12-6.

13. Li JJ, Nie SP, Qian XW, Zeng HS, Zhang CY. Chronic inflammatory status in patients with coronary artery ectasia. Cytokine 2009; 46: 61-4.

14. Işık T, Ayhan E, Uyarel H, et al. Association of neutrophil to lymphocyte ratio with presence of isolated coronary artery ectasia. Türk Kardiyol Dern Arş 2013; 41: 123-30.

15. Kundi H, Gok M, Kiziltunc E, et al. Relation between monocyte to high-density lipoprotein cholesterol ratio with presence and severity of isolated coronary artery ectasia. Am J Cardiol 2015; 116: 1685-9.

16. Manginas A, Cokkinos DV. Coronary artery ectasias: imaging, functional assessment and clinical implications. Eur Heart J 2006; 27: 1026-31.

17. Turhan $\mathrm{H}$, Erbay AR, Yasar AS, et al. Plasma soluble adhesion molecules; intercellular adhesion molecule-1, vascular cell adhesion molecule-1 and E-selectin levels in patients with isolated coronary artery ectasia. Coron Artery Dis 2005; 16: 45-50.

18. Aydin M, Tekin IO, Dogan SM, et al. The levels of tumo necrosis factor-alpha and interleukin- 6 in patients with isolated coronary artery ectasia. Mediators Inflamm 2009; 2009: 106145.

19. Turhan H, Erbay AR, Yasar AS, Balci M, Bicer A, Yetkin E. Comparison of C-reactive protein levels in patients with coronary artery ectasia versus patients with obstructive coronary artery disease. Am J Cardiol 2004; 94: 1303-6.
20. Shimizu M, Okada T, Kobayashi S, et al. Multiple coronary aneurysms resulting from isolated coronary vsculitis in an elderly patient. Circ J 2003; 67: 637-9.

21. Kajinami K, Kasashima S, Oda Y, Koizumi J, Katsuda S, Mabuchi H. Coronary ectasia in familial hypercholesterolemia: a histopathological study regarding matrix metalloproteinases. Mod Pathol 1999; 12: 1174-80.

22. Yildirim N, Tekin IO, Dogan SM, et al. Expression of monocyte and lymphocyte adhesion molecules is increased in isolated coronary artery ectasia. Coron Artery Dis 2007; 18: 49-53.

23. Yang R, Chang Q, Meng X, Gao N, Wang W. Prognostic value of systemic immune-inflammation index in cancer: a meta-analysis. J Cancer 2018; 9: 3295-302.

24. Zhong JH, Huang DH, Chen ZY. Prognostic role of systemic immune-inflammation index in solid tumors: a systematic review and meta-analysis. Oncotarget 2017; 8: 75381-8.

25. Seo M, Yamada T, Morita T, et al. Prognostic value of systemic immune-inflammation index in patients with chronic heart failure. Eur Heart J 2018; 39: ehy564.P589.

26. Lapenna D, de Gioia S, Mezetti A, et al. Cigarette smoke, ferritin and lipid peroxidation. Am J Respir Crit Care Med 1995; 151: 431-5. 